\title{
OTIMIZAÇÃO ROBUSTA APLICADA AO ROTEAMENTO DE AERONAVES NO TRANSPORTE AÉREO DE PASSAGEIROS SOB DEMANDA $^{1}$
}

\author{
Rafael Campos $^{\mathrm{a} *}$, Aldair Alvarez ${ }^{\mathrm{a}}$, Pedro Munari ${ }^{\mathrm{a}}$ \\ ${ }^{a}$ Departamento de Engenharia de Produção \\ Universidade Federal de São Carlos -UFSCar, São Carlos-SP, Brasil \\ Artigo premiado SBPO 2019: Trabalho de Iniciação Científica - 1o Lugar \\ Recebido 25/09/2019, aceito 17/10/2019
}

\begin{abstract}
RESUMO
Neste artigo, aborda-se o roteamento de aeronaves motivado pelo caso real de uma empresa de transporte aéreo de passageiros, que oferece um serviço de voos sob demanda. O objetivo é propor uma abordagem de apoio à tomada de decisão que, além de considerar os requisitos operacionais específicos do caso em estudo, incorpore também as incertezas inerentes aos tempos de viagem, os quais são bastante críticos em aviação. A partir de uma formulação matemática baseada no problema de roteamento de veículos com janelas de tempo e frota heterogênea, propõe-se um modelo de Otimização Robusta que permite incorporar tais incertezas por meio de uma perspectiva de pior caso. O uso dessa estratégia provou-se vantajoso por não necessitar da escolha de uma distribuição de probabilidades para representação da situação real. Experimentos computacionais realizados com dados de um mês de operação da empresa indicam os benefícios de se adotar a abordagem proposta. Por meio do uso da Simulação de Monte Carlo foi possível verificar uma redução de risco significativa nas soluções robustas.
\end{abstract}

Palavras-chave: Roteamento de aeronaves, Problema de roteamento de veículos, Otimização robusta.

\begin{abstract}
In this paper, we address aircraft routing operations motivated by the real-world case faced by an airline company that offers on-demand flights for passenger transportation. The purpose is to aid the decision-making process that not only considers the operational requirements that are specific for the studied case, but also take into account the inherent uncertainties of travel times, which are critical in the aviation business. From a mathematical formulation based on the vehicle routing problem with time windows and heterogeneous fleet, we propose a Robust Optimization model that incorporates these uncertainties through a worst-case perspective. This strategy has shown to be advantageous because it does not require the choice of a probability distribution to represent the real-world situation. Computational experiments with data obtained from one-month operation of the company indicate the benefits of adopting the proposed approach. By using Monte Carlo Simulation we could verify a significant reduction in the risks related to the robust solutions.
\end{abstract}

Keywords: Aircraft routing, Vehicle routing problem, Robust optimization.

\footnotetext{
* Autor para correspondência. E-mail: rafael.ajudarte.campos@gmail.com

DOI: 10.4322/PODes.2019.010
}

${ }^{1}$ Todos os autores assumem a responsabilidade pelo conteúdo do artigo. 


\section{Introdução}

A relevância teórica e prática do Problema de Roteamento de Veículos (PRV), em especial no contexto de transporte e logística, torna-o um objeto de estudo de muito interesse (Toth e Vigo, 2014). Não é recente o uso das ferramentas da Pesquisa Operacional para a solução deste tipo de problema, sendo que os trabalhos pioneiros sobre o tema, publicados por Dantzig e Ramser (1959) e Balinski e Quandt (1964), já contam com mais de meio século de existência. Desde então, a literatura tem sido bastante ativa em relação às variantes deste problema (Laporte et al., 2013), fazendo com que os métodos quantitativos sejam atualmente suportes confiáveis para o processo de tomada de decisão. Tais estudos primam pelo desenvolvimento de modelos matemáticos e métodos de solução, buscando aproximar a modelagem às condições reais, como questões de variabilidade dos parâmetros (Sungur et al., 2008) e tipos diferentes de restrições (Irnich et al., 2014), e de forma mais eficiente.

Problemas de roteamento de veículos, em sua essência, visam desenvolver rotas para um conjunto de veículos, que partem de um ou mais depósitos, com o mínimo custo ao visitar um conjunto de clientes. Pode-se entender como visitas a entrega de produtos ou prestação de um determinado serviço que tais veículos realizam nos clientes. Para tornar o problema mais realista, é comum adicionar restrições que se assemelham às condições reais, como: limite de capacidade no veículo, janelas de tempo para atendimento no cliente, heterogeneidade da frota, variabilidade nos parâmetros de tempo e demanda, dentre outros (Irnich et al., 2014; Toth e Vigo, 2002). Destas citadas, as três últimas apresentam elevada relevância para o presente trabalho. As janelas de tempo podem ser entendidas como restrições adicionais ao problema em que cada cliente $i$ deve ser visitado dentro de uma janela de tempo definida por um intervalo $\left[w_{i}^{a}, w_{i}^{b}\right]$. Assim, o veículo não pode chegar após o instante de tempo $w_{i}^{b}$ e, caso chegue antes de $w_{i}^{a}$, o veículo deve esperar até este instante para iniciar o serviço. Neste tipo de problema, considera-se também o parâmetro $s_{i}$ que corresponde ao tempo de serviço no cliente $i$ (Kallehauge et al., 2005; Desaulniers et al., 2014). A heterogeneidade da frota significa que os veículos apresentam características próprias, como capacidade, tempo de viagem ou custo diferentes (Toth e Vigo, 2002).

A variabilidade em parâmetros do problema, seja em tempos de viagem ou demanda, é outro fator muito interessante a ser estudado quando se modela uma situação real visto que, nesta, muitas das informações são desconhecidas ou incertas até o momento da operação (Ordonez, 2010). Esta é a principal razão que torna as abordagens utilizando programação estocástica e otimização robusta tão relevantes. Ainda segundo Ordonez (2010), há uma diversidade de métodos para a solução de problemas estocásticos, destacando-se três: restrições de chance, a programação estocástica com recurso e a otimização robusta. O primeiro consiste em modelar uma restrição capaz de garantir que a solução esteja no percentil $\alpha$, selecionado pelo tomador de decisão, da distribuição acumulada conhecida do parâmetro aleatório, de modo que a solução seja factível $\alpha \%$ das vezes. O segundo, por sua vez, consegue combater esta variabilidade por meio do uso de ações de recurso, que podem por exemplo permitir que o veículo volte para o depósito para reabastecer caso ele não consiga suprir algum cliente na rota base, aquela que é formada ainda com os parâmetros desconhecidos usando o valor esperado ou outro método.

Já a otimização robusta, conforme considerada neste artigo, trata-se de uma abordagem que visa a otimização do pior caso dos parâmetros variáveis, deste modo protegendo-se completamente contra tal incerteza, mas podendo apresentar custos maiores em situações normais. Cabe ao tomador de decisão ponderar se a troca é válida (Ben-Tal e Nemirovski, 1999). Uma vantagem que este método apresenta em relação à restrição de chances é que não se precisa conhecer a distribuição da incerteza, algo que pode ser complicado em situações reais, bastando ter conhecimento do pior caso da incerteza. Nota-se, porém, que não é necessário proteger-se de todas incertezas, visto que isto poderia acarretar em custos elevadíssimos para se proteger de uma situação praticamente impossível, aquela onde todas as variáveis estariam simultaneamente no pior caso possível. Pode-se, então, optar por uma proteção parcial destas incertezas, por meio de um parâmetro chamado de budget de incerteza, o qual o tomador de decisão pode decidir de 
forma a buscar um melhor custo-benefício (Bertsimas e Sim, 2003). Esta robustez será tratada no parâmetro $\Gamma$ que será melhor detalhado no decorrer do artigo.

O objetivo deste artigo é propor uma contraparte robusta para o modelo determinístico proposto por Munari e Álvarez (2019), os quais abordam o PRV no contexto do transporte aéreo de passageiros sob demanda. Assim, tem-se o estudo de um caso real envolvendo o roteamento de aeronaves, que permite a realização de experimentos computacionais com dados reais fornecidos pela empresa. Para incorporar incertezas no modelo determinístico, utiliza-se a abordagem proposta por Munari et al. (2019), que não requer a aplicação de esquema de dualização comumente usado na literatura para obtenção de modelos de otimização robusta. Isso resulta em um modelo de otimização robusta com menos variáveis e restrições, quando comparado a abordagens que recorrem à dualização, sendo assim mais eficiente a sua resolução por meio de softwares de otimização de propósito geral.

O restante deste artigo é estruturado da seguinte forma. Na Seção 2, apresenta-se a caracterização do problema e a formulação matemática determinística proposta por Munari e Álvarez (2019). O modelo de otimização robusta para o caso estudado é descrito na Seção 3. Os resultados computacionais são apresentados na Seção 4 e, por fim, são discutidas as conclusões e perspectivas futuras na Seção 5.

\section{Caracterização do Problema e Formulação Determinística}

O modelo desenvolvido por Munari e Álvarez (2019) foi criado para dar apoio à tomada de decisão em uma empresa de transporte aéreo de passageiros sob demanda que opera principalmente na Europa e Ásia, em particular nos serviços com contrato de posse fracionada. Neste tipo de contrato, o cliente tem posse de uma parte do avião, pagando uma parcela do valor total da aeronave, além de uma taxa de manutenção mensal, o que lhe dá direito a um determinado número de horas de viagem anuais. Diferentemente das empresas de vôos comerciais tradicionais, em que existem rotas com horários pré-definidos, o cliente pode escolher a rota desejada e avisar a empresa com até quatro horas de antecedência, embora tipicamente os avisos sejam feitos dias antes. Cabe a empresa, garantir que uma aeronave estará disponível no aeroporto e horário desejados.

Devido a esta forma particular de atendimento à demanda, a principal preocupação da empresa em relação às rotas são os vôos de reposicionamento. Estes são aqueles em que as aeronaves voam sem passageiros para chegar ao aeroporto de início de alguma requisição, incorrendo em custos que não são repassados diretamente para o cliente. Para entender melhor este conceito tem-se a Figura 1 que exemplifica o funcionamento de uma rota de uma aeronave. Inicialmente, a aeronave encontra-se no aeroporto de Madrid, Espanha, e como sua primeira missão é atender um cliente em Lisboa, Portugal, executa-se um vôo de reposicionamento até o aeroporto em que o cliente se encontra. Em seguida, atende-se a requisição do cliente, transportando-o até Zurique, na Suíça. Faz-se, então, um outro vôo de reposicionamento para Viena, Áustria, com o intuito de se atender a próxima requisição, que leva o cliente de Viena para Paris, França. Por fim, atende-se a última requisição que parte de Paris em direção a Oslo, Noruega. Nota-se que como a aeronave já se encontrava no aeroporto de Paris, não houve a necessidade de um vôo de reposicionamento para a última requisição.

A aeronave usada para atender o cliente deve ser semelhante ou superior àquela que ele contratou, o que resulta na necessidade de modelar uma frota heterogênea. Deve-se notar que, quando se usa aeronaves de nível superior ao contratado pelo cliente, é comum um aumento do custo de viagem. Além da frota heterogênea, o modelo ainda considera janelas de tempo para as requisições, tanto de atendimento dos clientes quanto para manutenção da aeronaves, permitindo assim uma tolerância para atrasos e mudanças nos horários desses eventos.

$\mathrm{O}$ modelo é construído a partir de um conjunto $V$ de aeronaves, segmentadas em um conjunto de $P$ tipos ordenados, formando assim os subconjuntos $V_{p}$ de aeronaves do tipo $p$. $\mathrm{O}$ conjunto de requisições é dado por $R$ e este é composto por: requisição dummy 0 ; conjunto $L$ de 
Figura 1: Exemplo de rota de vôo de uma aeronave.

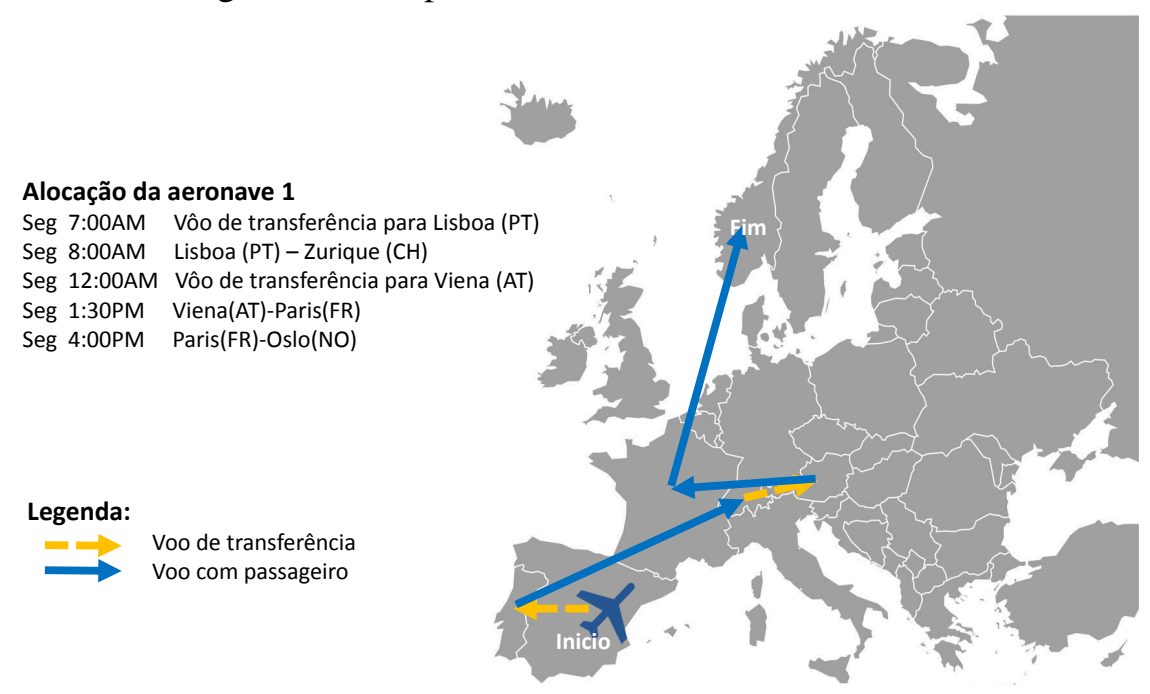

Fonte: Elaborada pelos autores.

pedidos do cliente; e conjunto de requisições de manutenção $M$. Os parâmetros relevantes para o modelo são $c_{v}$, representando o custo de voo por unidade de tempo da aeronave $v ; C_{v r s}$, entendido como o custo de reposicionamento da aeronave $v$ da requisição $r$ para a requisição $s$; $T_{i j}^{p}$ definido como o tempo de viagem entre os aeroportos $i$ e $j$ para uma aeronave do tipo $p ; A V_{v}$ é o momento exato que a aeronave $v \in V$ fica disponível para o horizonte de planejamento e $k_{v}$ é entendido como o aeroporto inicial da aeronave $v ; t_{v} \in P$ é o tipo da aeronave $v ; T A T_{k}^{r}$ é o tempo que a aeronave leva no aeroporto por questões como o embarque de passageiros e taxiamento; $S T_{r}$ é o horário de início da requisição $r ; \Delta_{L}$ e $\Delta_{M}$ apresentam o máximo que se pode adiantar ou atrasar o atendimento da requisição do cliente ou da manutenção, respectivamente; $i_{r}$ e $j_{r}$ representa os aeroportos de origem e destino, respectivamente, da requisição $r ; p_{r}$ é o tipo de aeronave requirida para a requisição $r$; $T L_{r}$ é o tempo de manutenção da requisição $r \in M$; e $v_{r}$ é a aeronave que deve passar por manutenção em uma requisição $r \in M$.

Figura 2: Representação do fluxo entre requisições no modelo.

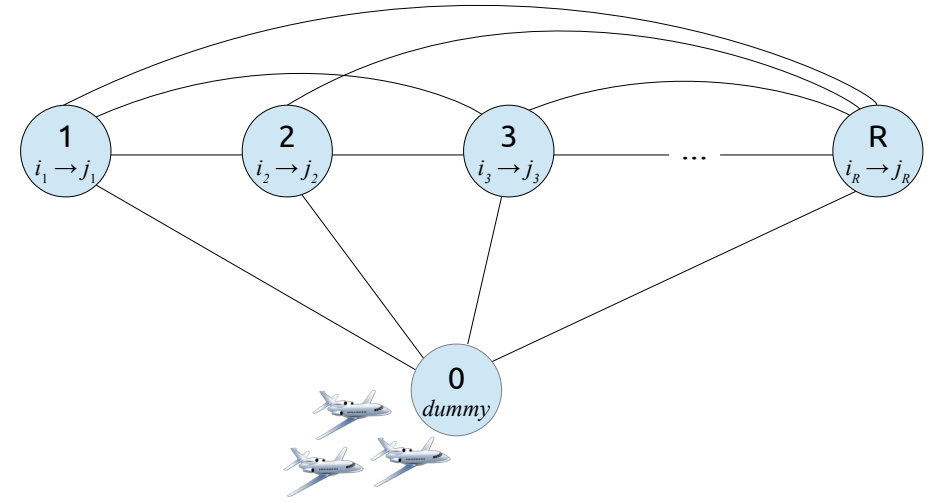

Fonte:Munari e Álvarez (2019).

Uma particularidade deste modelo é que ao invés de usar uma representação por uma rede em que os nós são os aeroportos e as variáveis de decisão determinam quais arcos escolher, comum em formulações clássicas do PRV (Letchford e Salazar-González, 2015; Desaulniers et al., 2014), opta-se por uma representação em que os nós são as requisições a serem realizadas e as variáveis de decisão determinam a ordem que estas serão efetuadas. Esta representação é exemplificada 
na Figura 2. Munari e Álvarez (2019) citam que a representação escolhida apresentou resultados bastante superiores às formulações tradicionais em relação a tempos computacionais. Esta mesma representação apresentou desempenho superior em uma formulação usada no contexto do transporte rodoviário de cargas (Munari et al., 2018).

Deste modo, as variáveis de decisão do problema são $y_{v r s}$, uma variável binária que assume valor $1 \mathrm{se}$, e somente se, a aeronave $v$ executa a requisição $s$, imediatamente após executar a requisição $r$; e $w_{r}$, uma variável contínua que representa o horário mais cedo que a requisição $r$ poderá ser executada. O modelo é então dado por:

$$
\begin{aligned}
& \min \sum_{v \in V} \sum_{r \in R} \sum_{s \in R} C_{v r s} y_{v r s}+\sum_{r \in L} \sum_{\substack{s \in R \\
s \neq r}} \sum_{\substack{p \in P \\
p>p_{r}}} \sum_{v \in V_{p}}\left(c_{t_{v}} T_{i_{r} j_{r}}^{t_{v}}-c_{p_{r}} T_{i_{r} j_{r}}^{p_{r}}\right) y_{v r s} \\
& \text { s.a } \sum_{\substack{p \in P \\
p \geq p_{r}}} \sum_{v \in V_{p}} \sum_{\substack{s \in R \\
s \neq r}} y_{v r s}=1 \\
& \sum_{\substack{s \in R \\
s \neq r}} y_{v_{r} r s}=1 \\
& \sum_{\substack{s \in R \\
s \neq r}} y_{v r s}=\sum_{\substack{s \in R \\
s \neq r}} y_{v s r} \\
& \sum_{s \in R} y_{v 0 s}=1=\sum_{r \in R} y_{v r 0}, \\
& S T_{r} \leq w_{r} \leq S T_{r}+\Delta_{L}, \\
& S T_{r}-\Delta_{M} \leq w_{r} \leq S T_{r}+\Delta_{M}, \\
& r \in L \\
& w_{s} \geq w_{r}+\sum_{v \in V}\left(T_{i_{r} j_{r}}^{p_{r}}+T A T_{j r}^{r}+T_{j_{r} i_{s}}^{p_{s}}+T A T_{i_{s}}^{s}\right) y_{v r s}+M_{r s}^{1}\left(\sum_{v \in V} y_{v r s}-1\right), \\
& r \in L, s \in R, r \neq s, s>0, j_{r} \neq i_{s}, \\
& w_{s} \geq w_{r}+\sum_{v \in V}\left(T_{i_{r} j_{r}}^{p_{r}}+T A T_{j s}^{s}\right) y_{v r s}+M_{r s}^{2}\left(\sum_{v \in V} y_{v r s}-1\right) \\
& r \in L, s \in R, r \neq s, s>0, j_{r}=i_{s}, \\
& w_{s} \geq\left(A V_{v}+T_{k_{v} i_{s}}^{t_{v}}+T A T_{i_{s}}^{s}\right) y_{v 0 s}, \\
& s \in L, v \in V, k_{v} \neq i_{s}, \\
& w_{s} \geq\left(A V_{v}+T A T_{i_{s}}^{s}\right) y_{v 0 s}, \\
& s \in L, v \in V, k_{v}=i_{s} \text {, } \\
& w_{s} \geq w_{r}+T L_{r}+T_{j_{r} i_{s}}^{p_{r}}+T A T_{i_{s}}^{s}+M_{r}^{3} s\left(y_{v_{r} r s}-1\right) \text {, } \\
& r \in M, s \in R, r \neq s, s>0, j_{r} \neq i_{s}, \\
& w_{s} \geq w_{r}+T A T_{i_{s}}^{s}+M_{r}^{4} s\left(y_{v_{r} r s}-1\right), \\
& w_{s} \geq\left(A V_{v_{s}}+T_{k_{v_{s}} i_{s}}^{p_{s}}\right) y_{v_{s} 0 s}, \\
& r \in M, s \in R, r \neq s, s>0, j_{r}=i_{s}, \\
& s \in M, k_{v_{s}} \neq i_{s} \text {, } \\
& s \in M, k_{v_{s}}=i_{s}, \\
& r \in R \text {, } \\
& w_{s} \geq y_{v_{s} 0 s} A V_{v_{s}} \text {, } \\
& w_{r} \geq 0 \text {, } \\
& v \in V, s, r \in R
\end{aligned}
$$

Dado o atendimento de todas as requisições ser obrigatório, não é preciso adicionar seus respectivos custos à função objetivo e, deste modo, a função objetivo (1) visa minimizar somente os custos de reposicionamento de aeronaves, entendido como o custo do avião viajar sozinho para atender alguma requisição, representado pelo primeiro somatório, e o custo adicional de atender uma requisição com uma aeronave com uma qualidade melhor, e mais custosa, que a contratada, representado pelo segundo somatório. As restrições (2)-(4) garantem o atendimento das requisições e o sequenciamento dos voos de cada aeronave. As restrições (5) determinam que toda aeronave deve partir e retornar para a requisição dummy. O respeito às janelas de tempo das requisições de clientes e de manutenção são determinadas pelas restrições (6) e (7), respectivamente. O cálculo do tempo mínimo para se iniciar uma requisição de cliente $s$ é executada pelas restrições (8) e (9), sendo que a primeira é para a situação em que se necessita de reposicionamento 
para atender a requisição $s$ após a $r$, e por isso precisa-se computar este tempo de viagem adicional, e a segunda é usada quando o destino da requisição $r$ é o mesmo da origem $s$ e por isso precisa-se somente computar o tempo de viagem da missão $r$ de fato. As restrições (10) e (11) calculam o horário mínimo que cada aeronave tem para atender a primeira requisição $r$ do período, sendo que o primeiro é usado quando o aeroporto inicial da requisição difere do aeroporto inicial da aeronave e o segundo é usado quando o aeroporto é o mesmo. As restrições (12)-(15) são análogas às restrições (8)-(11) porém para requisições de manutenção. O principal diferencial destas é que o aeroporto inicial e final da requisição é o mesmo, e usa-se o tempo de manutenção $T L_{r}$ ao invés do tempo de viagem. O domínio das variáveis é determinado pelas restrições (16) e (17).

\section{Modelagem Robusta}

Conforme já mencionado, propõe-se neste artigo uma contraparte robusta para o modelo (1)-(17). Para isso, utiliza-se a abordagem proposta por Munari et al. (2019), que consiste em definir novas variáveis que permitem modelar o comportamento dos parâmetros incertos por meio da programação dinâmica. Diferentemente da metodologia tradicional, apresentada em Bertsimas e Sim (2004) e usada por Agra et al. (2012) e Gounaris et al. (2013), em que se dualiza as restrições com parâmetros incertos no intuito de linearizá-las, a abordagem usada aqui baseia-se na linearização de equações recursivas que determinam o pior caso para a realização dos parâmetros incertos por meio da lógica de programação dinâmica. Esta metodologia, segundo Munari et al. (2019), além de ser de mais simples compreensão, apresenta melhores resultados computacionais pois resulta em uma contraparte robusta com número significativamente menor de variáveis e restrições em relação às demais abordagens.

Convém, inicialmente, compreender as equações de programação dinâmica que conseguem garantir a robustez desejada. Entende-se por $A$ como o conjunto de $\operatorname{arcos}(i, j)$ possíveis, sendo que $i, j \in N$; $\Gamma$ é definido como o budget de incerteza, representando o número máximo de piores casos tomados simultaneamente. Define-se também $w_{v_{j} \gamma}$ como o instante mais cedo em que se pode iniciar o serviço no nó $v_{j}$ quando se ocorre $\gamma \leq \Gamma$ piores casos simultâneos. Entende-se como pior caso possível da viagem a situação em que o tempo de viagem $T_{i j}^{p_{r}}$ entre os nós $i$ e $j$ assume seu valor máximo $\bar{T}_{i j}^{p_{r}}+\hat{T}_{i j}^{p_{r}}$, sendo $\bar{T}_{i j}^{p_{r}}$ o valor nominal e $\hat{T}_{i j}^{p_{r}}$ desvio máximo referentes ao tempo de viagem entre $i$ e $j$. O conjunto de incertezas em relação ao tempo de viagem que relaciona, e limita, os piores casos para a abordagem robusta é uma representação poliedral bem conhecida pela literatura (Bertsimas e Sim, 2003; Ordonez, 2010) que será definida como $U^{t}$. Adaptando-a ao modelo estudado, tem-se:

$$
U^{t}=\left\{T \in R^{|A|} \mid T_{i j}^{p_{r}}=T_{i j}^{\bar{p}_{r}}+\hat{T}_{i j}^{p_{r}} \delta_{i j}, \sum_{(i, j) \in A} \delta_{i j} \leq \Gamma, 0 \leq \delta_{i j} \leq 1, \forall(i, j) \in A\right\},
$$

em que $\delta_{i j}$ é o percentual do desvio máximo $\hat{T}_{i j}^{p_{r}}$ que foi tomado. Este percentual é limitado pelo $\Gamma$ selecionado, uma vez que a soma dos percentuais não pode superar o $\Gamma$ estabelecido. É interessante notar que, como se estuda a situação de pior caso na modelagem robusta, os $\delta_{i j}$ tenderão a ser ou 1 ou 0 , visto que o atraso máximo ocorrerá quando os $\Gamma$ maiores desvios $\hat{T}_{i j}^{p_{r}}$ estiverem integrais. Esta característica foi importante para os autores desenvolvessem a abordagem recursiva.

Denotando-se por $w_{j}^{a}$ e $w_{j}^{b}$ o instante de tempo mínimo e máximo das janelas de tempo do nó $j$, respectivamente, e por $s_{i}$ o tempo de serviço no nó $i$, tem-se a seguinte equação recursiva:

$$
w_{v_{j} \gamma}= \begin{cases}w_{v_{0}}^{a}, & \text { caso } j=0, \\ \max \left\{w_{v_{j}}^{a}, w_{v_{j-1} \gamma}+s_{v_{j-1}}+T_{v_{j-1} v_{j}}^{p_{r}}\right\}, & \text { caso } \gamma=0, \\ \max \left\{w_{v_{j}}^{a}, w_{v_{j-1} \gamma}+s_{v_{j-1}}+T_{v_{j-1} v_{j}}^{p_{r}},\right. & \\ \left.w_{v_{j-1} \gamma-1}+s_{v_{j-1}}+T_{v_{j-1} v_{j}}^{p_{r}}+\hat{T}_{v_{j-1} v_{j}}^{p_{r}}\right\}, & \text { caso contrário. }\end{cases}
$$

As duas primeiras linhas desta função são condições de contorno e a terceira verifica se o pior caso para o instante mais cedo de início do serviço é dado pelo horário de abertura da janela de 
tempo; ou tomando os $\gamma$ piores casos anteriores, sem utilizar o nó atual; ou usando os $\gamma-1$ piores casos anteriores e usando o pior caso do nó atual. A factibilidade da rota, na situação robusta determinada, será dada caso $w_{v_{j} \Gamma} \leq w_{v_{j}}^{b}$ para qualquer nó $j$. Se a condição for verdadeira indica que o horário mínimo para se começar o serviço em $j$ será menor que o horário máximo permitido pela janela de tempo para aquela robustez.

A ideia por trás dessa abordagem é converter as equações recursivas definidas em restrições para um modelo de programação linear inteira mista, que são similares às restrições comumente utilizadas para controle das variáveis de tempo. As restrições resultantes para um modelo geral com os parâmetros apresentados é o seguinte:

$$
\begin{array}{cl}
w_{j \gamma} \geq w_{i \gamma}+\left(s_{i}+T_{i j}^{p_{r}}\right) x_{i j}-M_{i j}\left(1-x_{i j}\right), & \forall(i, j) \in A, \gamma=0, \ldots, \Gamma \\
w_{j \gamma} \geq w_{i \gamma-1}+\left(s_{i}+T_{i j}^{p_{r}}+\hat{T}_{i j}^{p_{r}}\right) x_{i j}-M_{i j}\left(1-x_{i j}\right), & \forall(i, j) \in A, \gamma=1, \ldots, \Gamma \\
w_{i}^{a} \leq w_{i \gamma} \leq w_{i}^{b}, & \forall i \in N, \gamma=0, \ldots, \Gamma .
\end{array}
$$

As restrições (18) e (19) atuam de maneira similar à função recursiva, verificando para cada $\gamma$ e em um determinado nó $j$ se o tempo de início do serviço é maior se tomar os piores $\gamma$ casos dos nós anteriores, sem o pior caso do arco $(i, j)$, ou tomando os piores $\gamma-1$ casos anteriores e usando o pior caso do arco $(i, j)$. As restrições (20) garantem o respeito às janelas de tempo do modelo.

Deste modo, é possível adaptar o modelo (1)-(17) para um problema robusto que considera variabilidade no tempo de viagem. A função objetivo (1) e as restrições (2)-(5) e (17) permanecem exatamente as mesmas, visto que estas não dependem diretamente de $w_{r}$. As restrições (6), (7), (11), (13), (15) e (16) apenas sofrerão alterações no índice de $w_{r}$, que passará para $w_{r \gamma}$ uma vez que não há o parâmetro de tempo de viagem nestas restrições. Já as restrições (8), (9), (10), (12) e (14) sofrerão alterações mais profundas.

Tais mudanças serão exemplificadas mais detalhadamente pela restrição (8), por esta apresentar maior complexidade que as outras ao ter dois tempos de viagem. Neste caso, será necessário verificar em quais das situações o valor de $w_{r \gamma}$ é maior: usar $\gamma$ piores casos e o tempo de viagem normal para ambos tempos de viagem, representado por (21); usar $\gamma-1$ piores casos e considerar atraso somente na viagem da requisição, representado por (22); usar $\gamma-1$ piores casos e considerar atraso somente para a viagem de reposicionamento, representado por (23); ou usar $\gamma-2$ piores casos e considerar atraso em ambos tempos de viagem, representado por (24). É interessante notar que, para fins de modelagem, optou-se pela independência entre a variabilidade dos parâmetros. A restrição (8) pode ser reescrita, para todo $r \in L, s \in R, r \neq s, s>0, j_{r} \neq i_{s}$, como:

$$
\begin{aligned}
& w_{s \gamma} \geq w_{r \gamma}+\sum_{v \in V}\left(T_{i_{r} j_{r}}^{p_{r}}+T A T_{j r}^{r}+T_{j_{r} i_{s}}^{p_{s}}+T A T_{i_{s}}^{s}\right) y_{v r s} \\
& +M_{r s}^{1}\left(\sum_{v \in V} y_{v r s}-1\right), \quad \gamma \leq \Gamma \\
& w_{s \gamma} \geq w_{r(\gamma-1)}+\sum_{v \in V}\left(T_{i_{r} j_{r}}^{p_{r}}+\hat{T}_{i_{r} j_{r}}^{p_{r}}+T A T_{j r}^{r}+T_{j_{r} i_{s}}^{p_{s}}+T A T_{i_{s}}^{s}\right) y_{v r s} \\
& +M_{r s}^{1}\left(\sum_{v \in V} y_{v r s}-1\right), 0<\gamma \leq \Gamma(22) \\
& w_{s \gamma} \geq w_{r(\gamma-1)}+\sum_{v \in V}\left(T_{i_{r} j_{r}}^{p_{r}}+T A T_{j r}^{r}+T_{j_{r} i_{s}}^{p_{s}}+\hat{T}_{j_{r} i_{s}}^{p_{s}}+T A T_{i_{s}}^{s}\right) y_{v r s} \\
& +M_{r s}^{1}\left(\sum_{v \in V} y_{v r s}-1\right), 0<\gamma \leq \Gamma(23) \\
& w_{s \gamma} \geq w_{r(\gamma-2)}+\sum_{v \in V}\left(T_{i_{r} j_{r}}^{p_{r}}+\hat{T}_{i_{r} j_{r}}^{p_{r}}+T A T_{j r}^{r}+T_{j_{r} i_{s}}^{p_{s}}+\hat{T}_{j_{r} i_{s}}^{p_{s}}+T A T_{i_{s}}^{s}\right) y_{v r s} \\
& +M_{r s}^{1}\left(\sum_{v \in V} y_{v r s}-1\right), 1<\gamma \leq \Gamma \text {. }
\end{aligned}
$$


Com isto, pode-se aplicar o mesmo raciocínio para as restrições (9), (10), (12) e (14) de modo que, o modelo resultante será dado pela função objetivo (1) sujeito as restrições (2)-(5) e (17), unidas com (21)-(24) e:

$$
\begin{aligned}
& S T_{r} \leq w_{r \gamma} \leq S T_{r}+\Delta_{L} \\
& r \in L, \gamma \leq \Gamma \\
& S T_{r}-\Delta_{M} \leq w_{r \gamma} \leq S T_{r}+\Delta_{M}, \\
& r \in M, \gamma \leq \Gamma \\
& w_{s \gamma} \geq w_{r \gamma}+\sum_{v \in V}\left(T_{i_{r} j_{r}}^{p_{r}}+T A T_{j s}^{s}\right) y_{v r s}+M_{r s}^{2}\left(\sum_{v \in V} y_{v r s}-1\right), \\
& r \in L, s \in R, r \neq s, s>0, j_{r}=i_{s}, \gamma \leq \Gamma \\
& w_{s \gamma} \geq w_{r(\gamma-1)}+\sum_{v \in V}\left(T_{i_{r} j_{r}}^{p_{r}}+\hat{T}_{i_{r} j_{r}}^{p_{r}}+T A T_{j s}^{s}\right) y_{v r s}+M_{r s}^{2}\left(\sum_{v \in V} y_{v r s}-1\right), \\
& r \in L, s \in R, r \neq s, s>0, j_{r}=i_{s}, 0<\gamma \leq \Gamma, \\
& w_{s \gamma} \geq\left(A V_{v}+T_{k_{v} i_{s}}^{t_{v}}+\hat{T}_{k_{v} i_{s}}^{t_{v}}+T A T_{i_{s}}^{s}\right) y_{v 0 s}, \quad s \in L, v \in V, k_{v} \neq i_{s}, \gamma \leq \Gamma, \\
& w_{s \gamma} \geq\left(A V_{v}+T A T_{i_{s}}^{s}\right) y_{v 0 s}, \quad s \in L, v \in V, k_{v}=i_{s}, \gamma \leq \Gamma \text {, } \\
& w_{s \gamma} \geq w_{r \gamma}+T L_{r}+T_{j_{r} i_{s}}^{p_{r}}+T A T_{i_{s}}^{s}+M_{r}^{3} s\left(y_{v_{r} r s}-1\right), \\
& r \in M, s \in R, r \neq s, s>0, j_{r} \neq i_{s}, \gamma \leq \Gamma \\
& w_{s \gamma} \geq w_{r(\gamma-1)}+T L_{r}+T_{j_{r} i_{s}}^{p_{r}}+\hat{T}_{j_{r} i_{s}}^{p_{r}}+T A T_{i_{s}}^{s}+M_{r}^{3} s\left(y_{v_{r} r s}-1\right), \\
& r \in M, s \in R, r \neq s, s>0, j_{r} \neq i_{s}, 0<\gamma \leq \Gamma \\
& w_{s \gamma} \geq w_{r \gamma}+T A T_{i_{s}}^{s}+M_{r}^{4} s\left(y_{v_{r} r s}-1\right) \text {, } \\
& r \in M, s \in R, r \neq s, s>0, j_{r}=i_{s}, \gamma \leq \Gamma, \\
& w_{s \gamma} \geq\left(A V_{v_{s}}+T_{k_{v_{s} i_{s}}}^{p_{s}}\right) y_{v_{s} 0 s}, \\
& s \in M, k_{v_{s}} \neq i_{s}, \gamma \leq \Gamma \text {, } \\
& w_{s \gamma} \geq\left(A V_{v_{s}}+T_{k_{v_{s}} i_{s}}^{p_{s}}+\hat{T}_{k_{v_{s}} i_{s}}^{p_{s}}\right) y_{v_{s} 0 s}, \\
& s \in M, k_{v_{s}} \neq i_{s}, 0<\gamma \leq \Gamma \\
& w_{s \gamma} \geq y_{v_{s} 0 s} A V_{v_{s}} \text {, } \\
& s \in M, k_{v_{s}}=i_{s}, \gamma \leq \Gamma \\
& r \in R, \gamma \leq \Gamma \text {. }
\end{aligned}
$$

\section{Resultados Computacionais}

Nesta seção, são apresentados os resultados computacionais dos modelos estudados. Todos os testes foram executados em um computador com processador Intel Core i7-4790 CPU 3.60 $\mathrm{GHz}$ e com 16 GB de memória RAM, usando o software livre e gratuito GLPK 4.32. A decisão pelo uso de um software gratuito é compatível com o contexto real, em que a empresa optou por não investir em uma licença de software comercial.

Estudou-se os resultados para os dados de um mês da empresa, o terceiro mês das instâncias usadas em Munari e Álvarez (2019). Não se considerou nos experimentos do presente artigo, a possibilidade de upgrade, ou seja, atender o cliente com uma aeronave de nível superior à contratada. Cada instância, coletada por Munari e Álvarez (2019), cobre três dias consecutivos de dados reais. O uso de três dias para o horizonte de planejamento é compatível com aquele recomendado pela empresa. Com as informações reais, criou-se um conjunto de dados adaptado que leva em consideração um parâmetro que representa a variação máxima do tempo, estritamente positiva, de todas as viagens. Escolheu-se, de maneira arbitrária, uma variabilidade máxima de $10 \%, 25 \%$ e $50 \%$ em relação ao valor esperado.

Além disto, determinou-se o número máximo de piores casos que cada instância robusta poderia tomar, $\Gamma$, em $0,1,5$ e 10 . Nota-se que quando $\Gamma$ é 0 o modelo equivale a um problema determinístico. Em seguida, aplicou-se o modelo com todas combinações de $\Gamma$ e variabilidade. Os resultados encontram-se na Tabela 1. Nesta, apresenta-se o resultado médio das instâncias do mês estudado, em que SI é o resultado médio das soluções inteiras encontradas, Tempo é o tempo médio, em segundos, que os modelo levaram para processar a instância, $P d R$ é o preço da robustez, 
dado em porcentagem, entendido como o custo adicional que a instância robusta tem em relação à determinística. Originalmente, limitou-se o tempo de execução em 3600 segundos, porém todas as instâncias foram solucionadas até a otimalidade dentro deste limite e, portanto, os resultados correspondem a soluções ótimas.

Tabela 1: Resultado médio para um mês de dados com variabilidade de 10\%, $25 \%$ e $50 \%$.

\begin{tabular}{rccccccccc}
\hline & \multicolumn{3}{c}{$10 \%$} & \multicolumn{3}{c}{$25 \%$} & \multicolumn{3}{c}{$50 \%$} \\
\cline { 2 - 9 } & SI & PdR & Tempo(s) & SI & PdR & Tempo(s) & SI & PdR & Tempo(s) \\
\hline 0 & $162.726,8$ & - & 2,58 & $162.726,8$ & - & 2,58 & $162.726,8$ & - & 2,58 \\
1 & $165.904,7$ & $1,95 \%$ & 2,71 & $284.251,8$ & $74,68 \%$ & 2,71 & $296.077,2$ & $81,95 \%$ & 2,73 \\
5 & $167.238,0$ & $2,77 \%$ & 2,74 & $284.251,8$ & $74,68 \%$ & 2,82 & $312.577,8$ & $92,09 \%$ & 2,76 \\
10 & $167.238,0$ & $2,77 \%$ & 2,88 & $292.156,8$ & $79,54 \%$ & 2,89 & $312.577,8$ & $92,09 \%$ & 2,82 \\
\hline
\end{tabular}

Fonte: Elaborada pelos autores.

Com os dados da Tabela 1, é possível constatar que, para uma mesma variabilidade, os custos tendem a aumentar conforme o aumento de $\Gamma$. Isto é esperado visto o aumento dos tempos de viagem no sistema, resultando em sacrificar a otimalidade da solução nominal para garantir uma solução mais robusta. Por exemplo, com uma variabilidade de $10 \%$, os custos tendem a aumentar em 1,95\% em relação ao resultado determinístico, quando $\Gamma=1$, e 2,77\% para $\Gamma=5$ e $\Gamma=10$. Percebe-se também que o tempo computacional eleva-se conforme o aumento de $\Gamma$, porém este aumento é praticamente irrelevante visto que as soluções são alcançadas em até 3 segundos em média. Como as instâncias estudadas apresentam, em média, 25 veículos e 45 requisições, a eficiência computacional do modelo é bastante adequada para uso na situação real. É válido, ainda, notar que conforme se aumenta a variabilidade considerada nos tempos de viagem, o custo cresce vertiginosamente. Isto está relacionado ao maior risco de atrasos que acarreta em uma redução do número de requisições que cada aeronave poderá cumprir, resultando em rotas mais onerosas mas mais seguras. Além disso, mostra a criticidade dos atrasos no mercado de aviação.

Para avaliar o risco destas soluções na prática, realizou-se experimentos usando Simulação de Monte Carlo. Para realizar a simulação, 1000 amostras diferentes e uniformemente distribuídas de cada instância foram geradas para cada variabilidade e, em seguida, verificou-se se a solução robusta resolvia a instância sem violar as exigências das janelas de tempo para qualquer requisição. Os resultados encontram-se na Tabela 2, em que $P d R$ é novamente o preço da robustez, dado em porcentagem, e Risco é a porcentagem média de falhas que os modelos apresentaram para cada $\Gamma$ em cada variabilidade.

Tabela 2: Resultado das Simulações de Monte Carlo (média das instâncias estudadas).

\begin{tabular}{rcccccc}
\hline \multirow{2}{*}{$\Gamma$} & \multicolumn{2}{c}{$10 \%$} & \multicolumn{2}{c}{$25 \%$} & \multicolumn{2}{c}{$50 \%$} \\
\cline { 2 - 6 } & Risco & PdR & Risco & PdR & Risco & PdR \\
\hline 0 & $1,6 \%$ & - & $100 \%$ & - & $100 \%$ & - \\
1 & $0,3 \%$ & $1,95 \%$ & $0,9 \%$ & $74,68 \%$ & $34,2 \%$ & $81,95 \%$ \\
5 & $0 \%$ & $2,77 \%$ & $0,9 \%$ & $74,68 \%$ & $0,9 \%$ & $92,09 \%$ \\
10 & $0 \%$ & $2,77 \%$ & $0 \%$ & $79,54 \%$ & $0,9 \%$ & $92,09 \%$ \\
\hline \multicolumn{3}{c}{ Fonte: Elaborada pelos autores. }
\end{tabular}

Como os resultados indicam, conforme $\Gamma$ cresce, os custos se elevam, mas os riscos se reduzem de forma significativa. Com isso, observa-se a importância do tomador de decisão escolher um ponto compatível com o nível de serviço desejado e a variabilidade do sistema. Uma decisão excessivamente conservadora pode acarretar em elevados custos para a organização, enquanto uma decisão que considere pouco a variação pode resultar em um número excessivo de falhas ou atrasos, o que por sua vez é prejudicial para a imagem da empresa e pode levar a elevadas 
multas. O modelo visa, portanto, atuar como ferramenta de apoio à decisão, possibilitando encontrar rapidamente diversas soluções robustas e verificar seus custos e riscos associados.

As Figuras 3 e 4 apresentam, respectivamente, os resultados do modelo determinístico e do robusto, $\operatorname{com} \Gamma=1$, para a instância que abrange os dias 2 a 4 do mês estudado com variabilidade de $25 \%$. Nota-se que cada solução contém as rotas para cada uma das 27 aeronaves, então, para simplificar a comparação, as figuras somente apresentam as rotas que tivereram alterações entre as soluções determinísticas e robustas. As diferenças encontradas foram a necessidade de se reposicionar a aeronave da Inglaterra para atender o cliente no Alasca na rota robusta, ao invés de atendê-lo com a aeronave que atende a requisição que parte dos Estados Unidos em direção ao Alasca, e a necessidade de reposicionar a aeronave que executa o trajeto entre a Suíça e França para atender a requisição que parte da Inglaterra em direção a Suíça, ao invés de aproveitar a aeronave que executa a requisição que chega na Inglaterra partindo da Ucrânia. Estes reposicionamentos adicionais fazem com que a solução robusta apresente um valor $87,65 \%$ maior que a solução determinística. Por outro lado, o risco da solução robusta foi nulo, enquanto a solução determinística falhou em todas as amostras.

Figura 3: Solução determinística da instância que abrange os dias 2 a 4.

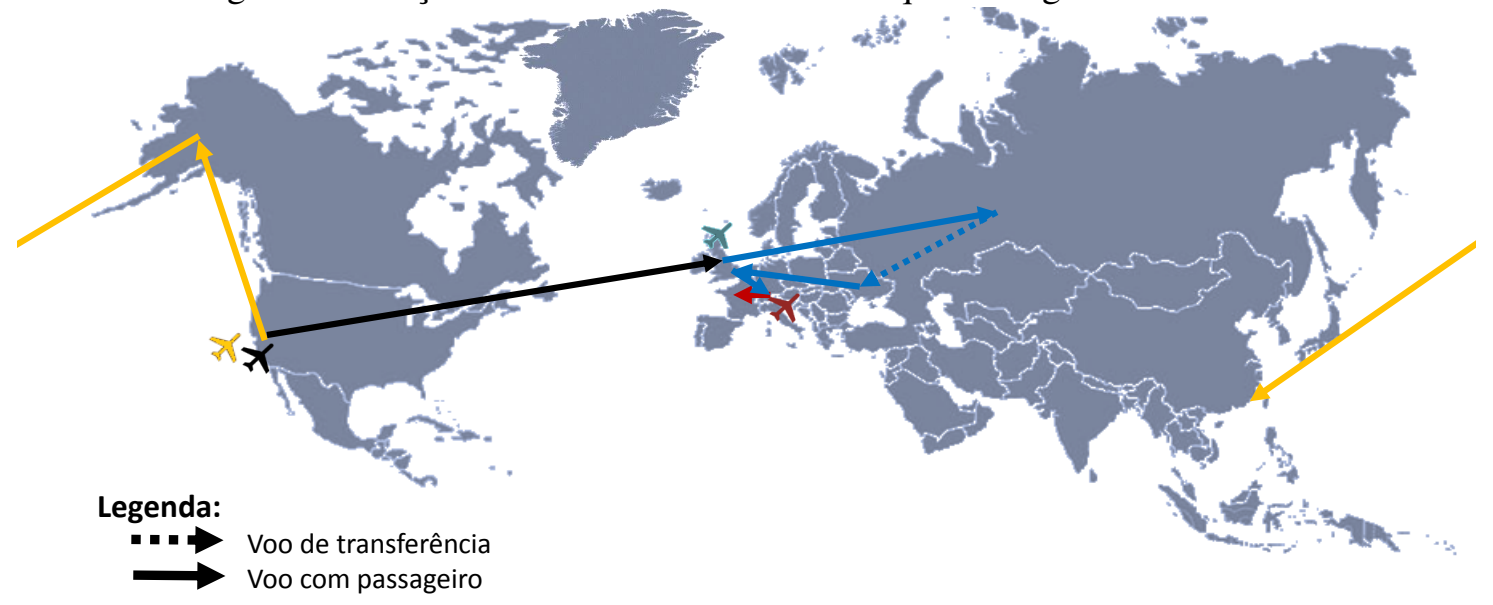

Fonte: Elaborada pelos autores.

\section{Conclusões}

Neste artigo, abordou-se o roteamento de aeronaves aplicado a um caso real de uma empresa de transporte aéreo de passageiros sob demanda. Foi proposto um modelo de otimização robusta que permitiu incorporar incertezas em relação a tempos de viagem, além de considerar os requisitos práticos da situação abordada. Para isso, foi utilizada uma abordagem que lineariza as equações recursivas que modelam o comportamento de pior caso dos parâmetros incertos, usando a lógica de programação dinâmica. O modelo proposto foi testado com dados reais, com diferentes valores de variabilidades baseados no tempo de viagem nominal. As soluções ótimas do modelo foram obtidas de forma rápida, por meio de um software de otimização de propósito geral, gratuito e de código livre. Fez-se, também, uso da Simulação de Monte Carlo para analisar os riscos das soluções de forma a servir como apoio a tomada de decisão ao apresentar os custos e os níveis de serviço de cada solução.

Notou-se que, conforme esperado, aumentar o nível de robustez do modelo resulta em soluções com menores riscos, porém mais custosas. Esta relação é perceptível principalmente quando o sistema está sujeito à elevada variabilidade, em que soluções determinísticas são incapazes de apresentar soluções factíveis na prática. Deste modo, o uso do modelo é interessante por 
Figura 4: Solução robusta, com variabilidade de $25 \%$ e $\Gamma=1$, da instância que abrange

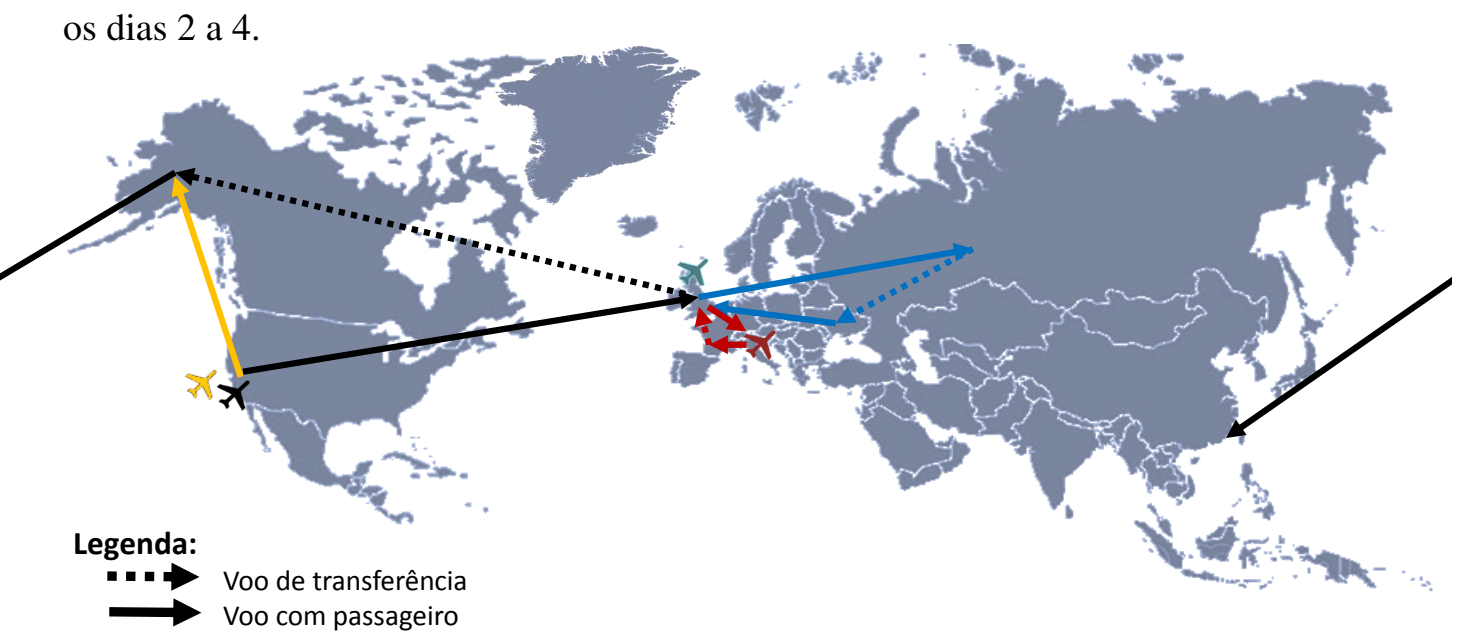

Fonte: Elaborada pelos autores.

permitir ao tomador de decisão avaliar as soluções considerando diferentes cenários e com diferentes níveis de robustez rapidamente, de modo que este pode escolher um ponto que considerar ótimo entre custo e risco.

Finalmente, como perspectiva para trabalho futuro, pretende-se realizar experimentos considerando a possibilidade de upgrade no atendimento de clientes, bem como usando instâncias de porte maior. Além disso, pretende-se incluir decisões de alocação de tripulação aos modelos descritos, uma decisão bastante crítica na prática, conforme levantado pela empresa que colaborou com este trabalho.

Agradecimentos. Os autores agradecem o apoio financeiro da Fundação de Amparo à Pesquisa do Estado de São Paulo (FAPESP), processos 2018/00463-4 , 2016/01860-1 e 2013/07375-0; do Conselho Nacional de Desenvolvimento Científico e Tecnológico (CNPq) e da Coordenação de Aperfeiçoamento de Pessoal de Nível Superior (CAPES).

\section{Referências}

Agra, A., Christiansen, M., Figueiredo, R., Magnus Hvattum, L., Poss, M., e Requejo, C. Layered formulation for the robust vehicle routing problem with time windows. In: International Symposium on Combinatorial Optimization, Berlin, Heidelberg. Springer, p. 249-260, 2012.

Balinski, M. L. e Quandt, R. E. On an integer program for a delivery problem. Operations Research, v. 12, n. 2, p. 300-304, 1964.

Ben-Tal, A. e Nemirovski, A. Robust solutions of uncertain linear programs. Operations Research Letters, v. 25, n.1, p. 1-13, 1999.

Bertsimas, D. e Sim, M. Robust discrete optimization and network flows. Mathematical Programming, v. 98, n. 1-3, p. 49-71, 2003.

Bertsimas, D. e Sim, M. The price of robustness. Operations Research, v. 52, n. 1, p. 35-53, 2004.

Dantzig, G. B. e Ramser, J. H. The truck dispatching problem. Management Science, v. 6, n. 1, p. 80-91, 1959. 
Desaulniers, G., Madsen, O. B., e Ropke, S. The vehicle routing problem with time windows. In: Toth, P. e Vigo, D. (ee.), Vehicle routing: Problems, methods, and applications, cap. 5, p. 119-159. MOS-SIAM Series on Optimization, 2014.

Gounaris, C. E., Wiesemann, W., e Floudas, C. A. The robust capacitated vehicle routing problem under demand uncertainty. Operations Research, v. 61, n. 3, p. 677-693, 2013.

Irnich, S., Toth, P., e Vigo, D. The family of vehicle routing problems. In: Toth, P. e Vigo, D. (ee.), Vehicle routing: Problems, methods, and applications, cap. 1, p. 1-33. MOS-SIAM Series on Optimization, 2014.

Kallehauge, B., Larsen, J., Madsen, O. B., e Solomon, M. M. Vehicle routing problem with time windows. In: Desaulniers, G., Desrosiers, J., e Solomon, M. M. (ee.), Column Generation, cap. 4, p. 67-98. Springer US, 2005.

Laporte, G., Toth, P., e Vigo, D. Vehicle routing: historical perspective and recent contributions. EURO Journal on Transportation and Logistics, v. 2, n. 1-2, p. 1-4, 2013.

Letchford, A. N. e Salazar-González, J.-J. Stronger multi-commodity flow formulations of the capacitated vehicle routing problem. European Journal of Operational Research, v. 244, n. 3, p. 730-738, 2015.

Munari, P., Alvarez, C., Bergamaschi, C., e Morabito, R. Um modelo de programação inteira mista baseado em rede de requisições para o problema de alocação de veículos. In: Anais do L Simpósio Brasileiro de Pesquisa Operacional, Rio de Janeiro. SOBRAPO, 2018. https://proceedings.science/p/85455.

Munari, P. e Álvarez, A. Aircraft routing for on-demand air transportation with service upgrade and maintenance events: Compact model and case study. Journal of Air Transport Management, v. 75 , p. $75-84,2019$.

Munari, P., Moreno, A., De La Vega, J., Alem, D., Gondzio, J., e Morabito, R. The robust vehicle routing problem with time windows: Compact formulation and branch-price-and-cut method. Transportation Science, v. 53, n. 4, p. 1043-1066, 2019.

Ordonez, F. Robust vehicle routing. In: Hasenbein, J. J., Gray, P. e Greenberg, H. J. (ee.) Risk and Optimization in an Uncertain World, cap. 7, p. 153-178. INFORMS, 2010.

Sungur, I., Ordonez, F., e Dessouky, M. A robust optimization approach for the capacitated vehicle routing problem with demand uncertainty. IIE Transactions (Institute of Industrial Engineers), v. 40 , n. 5, p. 509-523, 2008.

Toth, P. e Vigo, D. The Vehicle Routing Problem. SIAM, 2002.

Toth, P. e Vigo, D. Vehicle Routing: Problems, Methods and Applications. MOS-SIAM Series in Optimization, Second edition, 2014. 